\title{
Methods of Upper Body Training to Increase Overhand Throwing Power
}

\author{
Larry W. McDaniel \\ Department of Physical Education \& Exercise Science \\ Dakota State University \\ E-mail: larry.mcdaniel@dsu.edu \\ Allen Jackson \\ Department of Health, Physical Education \& Recreation \\ Chadron State College \\ E-mail: ajackson@csc.edu \\ Laura Gaudet \\ Department of Psychology, Counseling, \& Social Work \\ Chadron State College \\ E-mail: lgaudet@csc.edu
}

Shea Tonkin

Exercise Science

Dakota State University

\begin{abstract}
Those who have taught motor skills would most likely agree that the overhand throw may be the most difficult skill for students to master and for physical education instructors or coaches to teach. The timing involved when generating power to perform the overhand throw begins in the toes/feet and moves up the leg, hip, thorax (core muscles), arm, and ends in the finger tips. The overhand throw requires the coordination of most of the joints in the human body. Another consideration related to the complexity of the overhand throw is that the dominant or throwing side of the body is performing a completely opposite movement than the non-throwing side. The authors have analyzed the overhand throw joint by joint and then suggested methods of increasing power and strength in each area beginning with the fingers and ending in the muscles of the body's core. This paper provides information that suggests various methods of training for increasing power in the overhand throw by developing the core and upper body muscles. Often the overhand throw is performed without the assistance of force generated in the lower body. The focus of this paper is directed toward the development of upper body strength to compensate for situations where the lower body is in a position where it cannot contribution to the process of developing force when executing the overhand throw.
\end{abstract}

Keywords: Core muscles, Motor skills, Muscle analysis, Overhand throw, Rest intervals, Training methods, Upper body plyometric, Work intervals

\section{Introduction}

Those who have taught motor skills would most likely agree that the overhand throw may be the most difficult skill for students to master and for physical education instructors or coaches to teach. The timing involved when generating power to perform the overhand throw begins in the toes/feet and moves up the leg, hip, thorax, arm, and ends in the finger tips. The overhand throw requires the coordination of several joints in the human body. An additional consideration when teaching the overhand throw related to the complexity of the movement is that the dominant or throwing side of the body performs a completely opposite movement than the non-throwing side. However, the soccer kick may rival the overhand throw in some areas. When performing a soccer kick the intricate part of the skill involves balancing on one foot while striking the ball with the opposite foot. From the waist up the body is primarily involved in maintaining balance. A more difficult soccer kicking skill would be to maintain speed instead of slowing down or stopping when kicking the ball. The following information suggests various methods of training for increasing power in the overhand throw by developing the core and upper body muscles. Often the overhand throw is performed without the 
assistance of force generated in the lower body.

\section{Analysis of the Upper Body Muscles in the Overhand Throw}

The phases involved in the analysis of gross motor skill movements consist of the preparatory, force development, execution of the skill, and the follow through. When analyzing the right arm overhand throwing movement, the purpose of the preparatory phase (eccentric contraction) includes cocking the arm and maneuvering the joints on the right side of the body in the opposite direction of the throw. These movements are the foundation for all plyometric movements, a quick stretch (eccentric contraction) followed by a concentric contraction. The preparatory process aligns the body segments involved in the throw in a favorable position to not only generate and impart force but to facilitate accuracy (Luttgens \& Hamilton, 2006). The problem specific to the overhand throw is that often the thrower executes the skill without the luxury of maximum force generated by the lower body. In many gross motor skills the power to perform the skill is generated by a powerful push of the toes, the force developed by the foot, ankle, knee, and hip driving off the throwing surface. Additional power is generated by the core muscles (abdominals and obluques). The classic baseball catcher's stance is an excellent example. It is similar to a full squat with the buttock resting on the lower leg or calf muscles. Many times catchers snap off a quick throw from the squat position using primarily the force generated by the upper body. This paper will focus on the development of power in the upper body particular to overhand throwing.

\section{Fundamental Movement Analysis of the Core and Upper Body Muscles When Throwing}

The fundamental movements of the core muscles or trunk utilized in the overhand throwing motion includes flexion of the spine (Rectus Abdominis), rotation (Internal \& External Obliques), and lateral rotation of the trunk to the opposite side of the throwing arm (Internal \& External Obliques). While in the throwing motion, the upper arm movement involves the muscles that move the humerus of the shoulder joint (Anterior Deltoid, Pectoralis Major). The overhand throwing pattern produces medially rotation (Teres Major, Subscapularis, Deltoid, Pectoralis Major, \& Latissimus Dorsi) and extension of the humerus which resembles a whip-like movement of the arm (Latissimus Dorsi Teres Minor, \& Infraspinatus). The elbow joint contributes force by quick extension of the elbow (Triceps \& Anconeus). The wrist adds force by flexion and radial or ulnar flexion of the carpals (Flexor carpi Radialis \& Ulnaris). Flexion of the fingers is an important function in the last stage of imparting force on the object (Flexor Digitorum Profundus \& Superficialis). The phalanges impart force prior to the release of the object by flexing the fingers (Luttgens, K \& Hamilton N. 2006).

The training methods were divided into the following areas; fingers, hand, forearm, triceps, shoulder joint, and trunk. All of these areas may require unique training methods due to the demanding skills involved in the overhand throw. The exercises below may be used to develop power in the muscles involved in the joint movements analyzed previously in the throwing motion.

\section{Training Fingers, Hand, and Thumb Muscles}

When training the hand muscles, especially the thumb, start by squeezing a racquet ball or tennis ball with your thumb and fingers. This activity may increase thumb and finger strength. Increasing thumb and finger strength in the overhand throw may not only improve the skills related to grasping the object, but increase the amount of force generated in the throw. By squeezing a racquet ball or tennis ball, the number and severity of thumb and finger injuries may be reduced. An additional advantage of increasing thumb and finger strength is the improvement in hand and finger grasping while catching objects. These exercise's should be performed to failure with each hand alternating right and left hand exercises while letting the muscles in the free hand recover. These exercises may be completed on alternate days of the week.

\section{Training the Forearm and Wrist}

The first exercise recommended for training the forearm and wrist should be performed with an Olympic bar. Place the bar on your backside just below the buttocks, using an overhand grip, relax and contract the forearm by alternating flexion and extension of the wrist. Perform this exercise for three sets at 15-20 reps per/set. Wrist rollers that provide wrist flexion exercises may be effective for these athletes by reversing the direction of a weighted wrist roller to include wrist extension. The next exercise involves filling a bucket or large bowl with rice or kernels of corn and digging toward the bottom of the bucket alternating right and left hands. This exercise strengthens grasping movements. By opening and closing the hand, while digging in rice, the athlete will strengthen both the extensors and flexors of the fingers and thumb. Next, the athlete should post one forearm at a time on a bench while allowing the wrist and hand to extend past the support. The athlete should use a light dumbbell to perform the following exercises: First, with your hand in a neutral position mimic the movements of pounding a nail with a hammer isolating on the wrist action. This movement is ulnar and radial flexion which may be used in throwing and catching motions.

\section{Training Triceps Muscles}

The triceps is a major muscle in the throwing motion of the upper arm. Supine overhead triceps extensions, standing kickbacks, triceps pull downs with a rope, overhead dumbbell extensions, and close grip bench are exercises that 
strengthen the triceps. The triceps are an important muscle used in throwing. The thrower may not always have time to gather momentum with their legs and throw. The overhand throwing movement is sometimes modified to resemble a dart being thrown at a dart board. The above movement has now been modified into a short and quick movement in the arm, shoulder, and trunk. Standing and supine overhead throws with a medicine ball will activate many of the muscles involved in the upper body. The triceps may be even more stimulated if each overhead throw is started with the elbows flexed. When the focus is on strengthening the upper body of the athlete, it is important to remember to use the leg muscles as little as possible. The following is a list of plyometric exercises to develop upper body power.

\section{Medicine Ball Chest Pass}

Hold the medicine ball with both hands in front of your chest. Push-pass the ball to a partner, as in performing chest passes in basketball. The partner should immediately pass the medicine ball back to you upon catching the ball. Try to pass the ball quickly back and forth. Force is generated by horizontal adduction of the humerus, extension of the elbow, and flexion of the wrist and fingers. The core muscles of the trunk are in flexion when the ball is released (Reference \#1, \#4, \#6, \&\#11).

\section{Ballistic Pushups}

Ballistic pushups are simply pushups that launch the body off the ground with each repetition. Upon landing, immediately launch the body upward. Variations of this exercise include clapping hands in mid-air after pushing off the ground, or launching the body with enough force so that both hands and feet are airborne. Another alternative exercise involves a slight lateral movement with each repetition, so that the body traverses in a circle with the feet at the center of the circle spending as little time as possible in contact with the ground (see figure \#7 below).. Force is generated by horizontal adduction of the humerus, extension of the elbow, flexion of the wrist and fingers. The core muscles of the trunk are in a static state of contraction (Reference \# 7).

\section{Training the Trunk (Core Muscles)}

Core muscles of the trunk include the abdominal muscles, internal oblique, external oblique and back muscles (Erector Spinae). Overhand throws require the core muscles to contribute force to the movement when the legs are not a factor in generating force. Notice that plyometric exercises were selected over weight training exercises. When training the core muscles for power barbells, dumbbells, and weight plates may not be as safe. Medicine ball plyometric activities will be explained in detail below.

\section{Overhead Throws}

1. Stand with one foot in front (staggered stance) with knees slightly bent.

2. Move the medicine ball back behind the head and forcefully throw the ball forward as far as possible to a wall.

3. Catch the medicine ball on the bounce from the wall and repeat according to prescribed repetitions. Keep the time between pulling the ball back and starting the throw (transition phase) to a minimum. This exercise may also be performed with a partner.

Tip, when drawing the arms back in preparation for the throw, place the elbows in a flexed position. Force is generated by extension of the shoulder joint and elbow, flexion of the wrist and fingers, and flexion of spine (References \#1, \#4, \#6, \&\#11).

\section{Side Throws}

1. Stand with feet hip width apart placing the left foot approximately one foot in front of right.

2. Hold medicine ball with both hands with arms slightly bent.

3. Swing the ball over the right hip and forcefully underhand toss the medicine ball forward to a partner or wall maintaining abdominal muscle involvement to maximize proper usage of core muscles.

4. Catch the ball on the bounce from a partner or wall and repeat.

Force is generated by diagonal movements of the shoulder joint, flexion of the elbow, flexion and ulnar/radial flexion of the wrist, flexion of the fingers, while the spine rotations and lateral flexes (References \#3, \#8, \&\#13).

\section{Overhead Backward Throw}

1. Stand with feet slightly wider than the hips with a partner or trainer standing approximately 10-15 yards behind you.

2. Grasp the ball and lower ones' body into a semi-squat position. Explode upward extending the entire body releasing the medicine ball up and over the body.

3. The goal is to throw the ball behind you as far as possible generating most of the power with the legs. This movement may improve the coordination of the upper and lower body.

4. Catch the ball on the bounce from a partner and repeat according to prescribed repetitions. 
Force is generated by flexion of the shoulder joint, flexion of the elbow, wrist, and fingers while the spine extends (References \#10 below).

\section{Slams}

1. Stand with feet parallel approximately shoulder-width apart with the knees slightly bent.

2. Pull the medicine ball back behind your head and forcefully throw the ball down on the ground.

3. Catch the ball on the bounce and repeat according to prescribed repetitions.

Force is generated by extension of the shoulder joint and elbow, flexion of the wrist, flexion of the fingers, and flexion of spine (Reference \#4).

\section{Explosive Start Throws}

1. Stand with feet slightly wider than the hips. Knees should be slightly bent.

2. Pick the medicine ball up to chest level.

3. Quickly jump driving the body upward and simultaneously pressing the ball straight out as far and fast as possible.

4. As the ball is pressed forward, push off hard with either leg so that your body actually moves forward a couple of steps.

Force is generated by horizontal adduction of the shoulder joint, extension of the elbow, flexion of the wrist, flexion of the fingers, and flexion of spine (once an upright trunk position has been established).

\section{Single Arm Overhead Throws}

1. Stand with feet slightly wider than hip-width.

2. Grasp medicine ball and bend ankles, knees, and hips into a semi-squat position. Throw the medicine ball up in the air explode upward extending the entire body.

3. The goal is to throw the ball as high as possible generating most of the power in the legs coordinating the movements in the upper and lower body.

4. Catch the ball on the bounce and repeat.

Force is generated by abduction of the shoulder joint, extension of the elbow, flexion of the wrist, flexion of the fingers, and flexion of spine. (Reference\#11)

\section{Squat Throws}

1. Posture, stand with feet slightly wider than the wide of the hips with the knees should be slightly bent.

2. Hold a medicine ball at chest level and squat down to a parallel position.

3. Quickly explode upward out of the squat position and jump as high as possible. At the start of the jump, begin shoulder pressing the ball to full extension; finish the movement at the peak of the jump. End the movement by pushing the ball as high as possible into the air. It is important to minimize the time spent in the squat position by quicklying jump up as quickly as possible.

4. Catch the ball on the bounce and repeat according to the number of prescribed repetitions.

Force is generated by abduction of the shoulder joint, extension of the elbow, flexion of the wrist and fingers, and flexion of spine (Reference \#5).

\section{Tire Throw}

The tire throw is performed much like a discus throw. The movement starts by gripping the inside edge of the tire with one or both hands. Then rotate the trunk in the opposite direction of the throw keeping elbows slightly flexed and the lead arm shoulder height. The trail arm is approximately at hip height. Once the arm and tire are behind the body quickly rotate the trunk to the left (for throwing with the right arm) and release the tire at approximately a 45 degree angle in the direction of the throw. Force is developed by horizontal adduction of the shoulder joint, flexion of the elbow, ulnar flexion of the wrist, and flexion of the fingers. Prior to the release of the tire, rotation and lateral flexion of the spine to the opposite side is performed. (Martialarts.jameshom.com) (Reference \#3 -13).

When using the above exercises the following work to rest intervals may be used. Work to rest intervals of 1:5 to 1:15 and 3 sets of 3-6 reps are recommended for power activities. One to five (1:5) work to rest intervals are recommended for training the glycolytic system while 1-15 intervals are recommended for the ATP-PCr system. These metabolic systems may be enhanced by plyometric activities (Wilmore \& Costill, 2004).

\section{References}

Hall, S. B. (2007). Basic biomechanics. Boston, MA: McGraw-Hill. 
Luttgens, K \& Hamilton, N. (2006). Kinesiology: Scientific basis of human motion.

Boston, MA: McGraw-Hill. Martialarts.jameshom.com (http://www.martialarts.jameshom.com/)

Nordin, M. \& Frankel, V. (2001). Basic biomechanics of the musculoskeletal system. Baltimore, MD:Lippincott, Williams \& Williams Publishers.

Thompson, C. W. \& Floyd, R.T. (2001). Manual of structural kinesiology. Boston, MA: McGraw-Hill. Sporting Excellence. (n.d.). Upper body plyometric drills. Sport Fitness Advisor: Sports Training Tips for Athletic Peak Performance. Retrieved 2008, from http://www.sport-fitness-advisor.com/plyometric-drills.html.

Wilmore, J. H. \& Costill, D. L. (2004). Physiology of Sport \& Exercise. Champaign, IL: Human Kinetics.

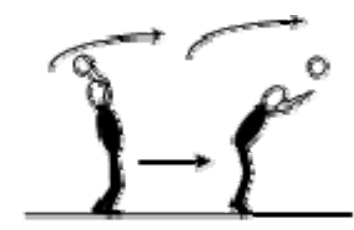

H1

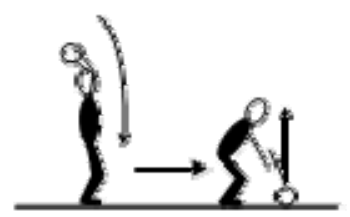

4

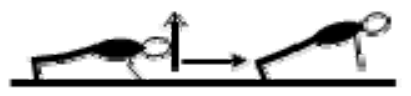

$n$

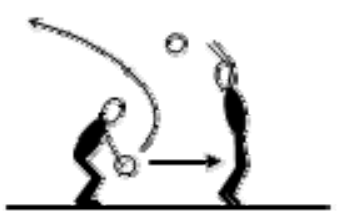

H10

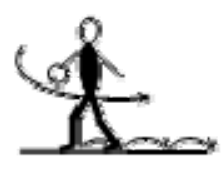

H13

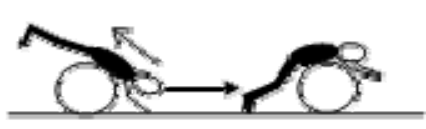

$n$

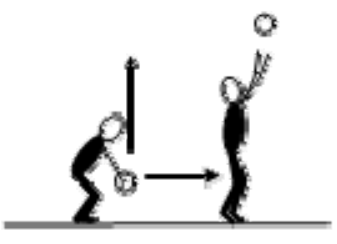

s

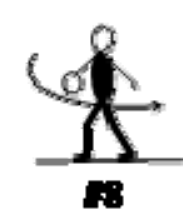

i.

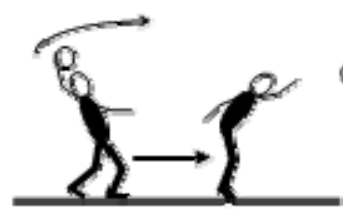

Al

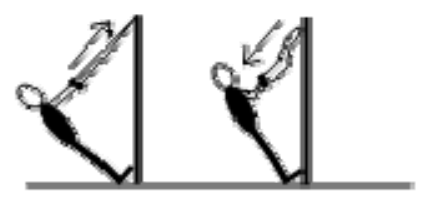

A4

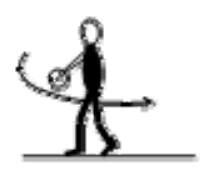

$\boldsymbol{B}$

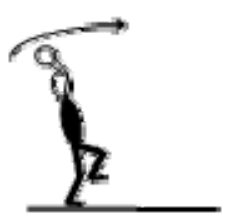

A6

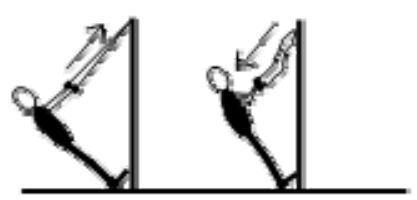

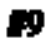

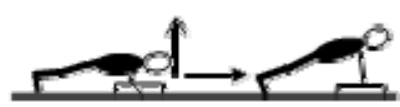

12

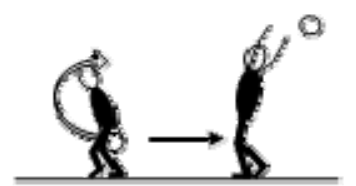

As

Figure 1. Upper Body Medicine Ball Activities

Source:http://www.performbetter.com/catalog/matriarch/OnePiecePage.asp_Q_PageID_E_60_A_PageName_E_Article Plyometrics3 